\title{
Velocity and Acceleration in Parabolic Coordinates
}

\author{
D. J Koffa ${ }^{1}$, J. F Omonile ${ }^{2}$, S. X. K Howusu ${ }^{2}$. \\ ${ }^{I}$ Department of Physics, Federal University Lokoja, Lokoja. Nigeria \\ ${ }^{2}$ Department of Physics, Kogi State University, Anyigba. Nigeria
}

\begin{abstract}
The expression for the velocity and acceleration in prolate spheroidal coordinates is now well known. In this paper, we proceed to derive expression for the instantaneous velocity and acceleration in Parabolic Coordinates for applications in Newtonian's Mechanics, Einstein's Special Law of Motion and Schrödinger's Law of Quantum mechanics.
\end{abstract}

Keywords: Acceleration, Newtonian's Mechanics, Parabolic coordinates, Velocity.

\section{Introduction}

Velocity and acceleration in prolate spheroidal coordinates had been established [1]. We therefore continue to derive the expression for velocity and acceleration in parabolic coordinates for application in mechanics. The parabolic coordinates $(\xi, \eta, \phi)$ are defined in terms of the Cartesian coordinates $(x, y, z)$ by [2]; $x=\xi \eta \cos \phi$

$y=\xi \eta \cos \phi$

$z=\frac{1}{2}\left(\eta^{2}-\xi^{2}\right)$

where

$0 \leq \xi<\infty ; 0 \leq \eta<\infty ; 0 \leq \phi \leq 2 \pi$

Consequently, by definition, the parabolic metrical coefficients are given by:

$h_{\xi}=\left(\xi^{2}+\eta^{2}\right)^{2}$

$h_{\eta}=\left(\xi^{2}+\eta^{2}\right)^{2}$

and

$h_{\phi}=\xi \eta$

These metrical coefficients define the units vectors, line element, volume element, gradient, divergence, curl and Laplacian operations in parabolic coordinates, according to the theory of orthogonal curvilinear coordinates $[2,3,4]$. These quantities are necessary and sufficient for the derivation of the fields of all parabolic distribution of mass, charge and current. Now for the derivation of the equations of motion for test particles in these fields, we shall derive the expression for instantaneous velocity and acceleration in parabolic coordinates.

\section{Mathematical Analysis.}

The Cartesian unit vectors are related to the parabolic coordinates unit vector as:

$$
\begin{aligned}
& \hat{\xi}=\frac{1}{\left(\xi^{2}+\eta^{2}\right)^{\frac{1}{2}}}[\eta \cos \phi \hat{l}+\eta \sin \phi \hat{\jmath}-\xi \hat{k}] \\
& \hat{\eta}=\frac{1}{\left(\xi^{2}+\eta^{2}\right)^{\frac{1}{2}}}[\xi \cos \phi \hat{l}+\xi \sin \phi \hat{\jmath}-\eta \hat{k}] \\
& \text { and } \\
& \hat{\phi}=-\cos \phi \hat{l}+\sin \phi \hat{\jmath}
\end{aligned}
$$

Hence denoting one time differentiating by a dot, it follows from (8), (9) and (10) and some manipulation that:

$$
\hat{\xi}=\frac{1}{\xi^{2}+\eta^{2}}[-\eta \dot{\xi}+\dot{\eta} \xi] \hat{\xi}+\frac{1}{\left(\xi^{2}+\eta^{2}\right)^{\frac{1}{2}}} \dot{\phi} \eta \hat{\phi}
$$

Similarly, it follows from (9), (8) and (10) that:

$\hat{\eta}=\frac{1}{\xi^{2}+\eta^{2}}[\dot{\xi} \eta-\xi \dot{\eta}] \hat{\xi}+\frac{1}{\left(\xi^{2}+\eta^{2}\right)^{\frac{1}{2}}} \dot{\phi} \xi \hat{\phi}$

and

consequently, from (10), (8) and (9):

$\hat{\phi}=\frac{-1}{\left(\xi^{2}+\eta^{2}\right)^{\frac{1}{2}}}[\eta \dot{\phi} \hat{\xi}+\xi \dot{\phi} \hat{\eta}]$

Now it follows from definition of instantaneous position vector $\underline{r}$, as

$\underline{r}=x \hat{l}+y \hat{\jmath}+z \hat{k}$ 
and $(8)-(10)$ that the instantaneous position vector may be expressed entirely in terms of parabolic coordinates as:

$\underline{r}=\frac{1}{2} \xi\left(\eta^{2}+\xi^{2}\right)^{\frac{1}{2}} \hat{\xi}+\frac{1}{2} \eta\left(\xi^{2}+\eta^{2}\right)^{\frac{1}{2}} \hat{\eta}$

It now follows from definition of instantaneous velocity vector, $\underline{u}$ as:

$\underline{u}=\underline{r}$

and (15), (11)-(13) that the instantaneous velocity vector may be expressed entirely in terms of parabolic coordinate as:

$\underline{u}=u_{\xi} \hat{\xi}+u_{\eta} \hat{\eta}+u_{\phi}$

where

$u_{\xi}=\left(\eta^{2}+\eta^{2}\right)^{\frac{1}{2}} \dot{\xi}$

$u_{\eta}=\left(\xi^{2}+\eta^{2}\right)^{\frac{1}{2}} \dot{\eta}$

and

$u_{\phi}=\xi \eta \dot{\phi}$

Similarly, it follows from definition of instantaneous acceleration, $\mathrm{g}$, as:

$\underline{a}=\underline{u}$

And (8)-(20) and (11)-(13) that the instantaneous acceleration may be expressed entirely in terms of parabolic coordinate as:

$\underline{a}=a_{\xi} \hat{\xi}+a_{\eta} \hat{\eta}+a_{\phi} \hat{\phi}$

where

$a_{\xi}=\left(\xi^{2}+\eta^{2}\right)^{\frac{1}{2}}\left[\ddot{\xi}+\frac{\xi \dot{\xi}^{2}}{\xi^{2}+\eta^{2}}-\frac{\xi \dot{\eta}^{2}}{\xi^{2}+\eta^{2}}-\frac{\xi \eta^{2} \dot{\phi}^{2}}{\xi^{2}+\eta^{2}}+\frac{2 \eta \dot{\xi} \dot{\eta}}{\xi^{2}+\eta^{2}}\right]$
$a_{\eta}=\left(\xi^{2}+\eta^{2}\right)^{\frac{1}{2}}\left[\ddot{\eta}+\frac{\eta \dot{\eta}^{2}}{\xi^{2}+\eta^{2}}-\frac{\eta \dot{\xi}^{2}}{\xi^{2}+\eta^{2}}-\frac{\xi^{2} \eta \dot{\phi}^{2}}{\xi^{2}+\eta^{2}}+\frac{2 \xi \dot{\eta} \dot{\xi}}{\xi^{2}+\eta^{2}}\right]$

and

$a_{\phi}=\xi \eta\left[\ddot{\phi}+\frac{2 \dot{\xi} \dot{\phi}}{\xi}+\frac{2 \eta \eta \dot{\phi}}{\eta}\right]$

This is the completion of the parabolic coordinates system.

\section{Results and Discussion}

In this paper we derived the component of velocity and acceleration in parabolic coordinates as (18)-(20) and (22)-(24) respectively. These results obtained in this paper are necessary and sufficient for expressing all mechanical quantities (Linear momentum, kinetic energy, Lagranian and Hamiltonian) in terms of parabolic coordinates.

\section{Conclusion}

The velocity and acceleration equations (18), (19), (20), (22), (23), (24) obtained in this paper paves a way for expressing all dynamical Laws of motion (Newton's Law, Lagrange's Law, Hamiltonian's Law, Einstein's Special Relativities Law of motion and Schrödinger's Law of quantum mechanics) entirely in terms of parabolic coordinates.

\section{References}

[2] S.X.K. Howusu, vector and tensor analysis (Jos: Jos University press Ltd, 2003).

[3] G. Arfken, Mathematical Methods for Physicists ( New-York: Academic press, 1968) 467-469.

[4] F.B. Hilderbrand, Advanced calculus for Applications ( Prentice Hall: Englewood-Cliff, 1962) $298-306$.

[5] Morce. H. Feshbach, Method of Theoretical Physics(New-York: Mc Graw-Hill, 1953) 21-54

[6] B. Garfinkel, Problem of Quadratures, Astron J. 63, 88, 1958 